\title{
Structure of a novel $\operatorname{lns} \mathrm{P}_{3}$ receptor
}

\section{Thomas C.Südhof, Christopher L.Newton, Branch T.Archer III, Yuri A.Ushkaryov and Gregory A.Mignery}

Department of Molecular Genetics and Howard Hughes Medical Institute, University of Texas Southwestern Medical Center, Dallas, TX 75235, USA

Communicated by J.Meldolesi

Inositol 1,4,5-trisphosphate $\left(\mathrm{InsP}_{3}\right)$ constitutes a major intracellular second messenger that transduces many growth factor and neurotransmitter signals. InsP $_{3}$ causes the release of $\mathrm{Ca}^{2+}$ from intracellular stores by binding to specific receptors that are coupled to $\mathrm{Ca}^{2+}$ channels. One such receptor from cerebellum has previously been extensively characterized. We have now determined the full structure of a second, novel InsP receptor which we refer to as type $2 \mathrm{InsP}_{3}$ receptor as opposed to the cerebellar type $1 \mathrm{InsP}_{3}$ receptor. The type 2 Ins $\mathrm{P}_{3}$ receptor has the same general structural design as the cerebellar type 1 InsP $\mathrm{P}_{3}$ receptor with which it shares $69 \%$ sequence identity. Expression of the aminoterminal 1078 amino acids of the type 2 receptor demonstrates high affinity binding of $\mathrm{InsP}_{3}$ to the type 2 receptor with a similar specificity but higher affinity than observed for the type 1 receptor. These results demonstrate the presence of several types of $\mathrm{InsP}_{3}$ receptor in brain and raise the possibility that intracellular $\mathrm{Ca}^{2+}$ signaling may involve multiple pathways with different regulatory properties dependent on different $\mathrm{InsP}_{\mathbf{3}}$ receptors.

Key words: $\mathrm{Ca}^{2+}$ channel/endoplasmic reticulum/ intracellular $\mathrm{Ca}^{2+} /$ ryanodine receptor/signal transduction

\section{Introduction}

The cellular responses to many growth factors and neurotransmitters is mediated by increases in intracellular $\mathrm{Ca}^{2+}$ caused by the release of InsP $\mathrm{P}_{3}$ (Berridge and Irvine, 1989). Ins $\mathrm{P}_{3}$ binds to specific intracellular receptors and causes the release of $\mathrm{Ca}^{2+}$ from intracellular $\mathrm{Ca}^{2+}$ stores that are probably part of the endoplasmic reticulum (Streb et al., 1983). Generation of intracellular $\mathrm{InsP}_{3}$ also leads to the delayed influx of $\mathrm{Ca}^{2+}$ via plasma membrane channels by an unidentified mechanism (Penner et al., 1988). In addition, InsP $\mathrm{P}_{3}$ is instrumental in establishing or maintaining $\mathrm{Ca}^{2+}$ oscillations in many cells (Woods et al., 1986; Berridge, 1990; Harootunian et al., 1991; Petersen et al., 1991).

An $\mathrm{InsP}_{3}$ receptor from cerebellum has been well characterized (reviewed in Ross et al., 1990; Shears, 1991) and immunolocalized to all parts of the endoplasmic reticulum in Purkinje cells (Mignery et al., 1989; Ross et al., 1989). The cerebellar InsP $\mathrm{P}_{3}$ receptor consists of a homotetramer of $M_{r} 313000$ subunits that are encoded by a $10 \mathrm{~kb}$ mRNA which is subject to at least two different alternative splicing events (Mignery et al., 1989, 1990; Furiuchi et al., 1989; Ferris et al., 1991a). The primary structure of the $\mathrm{InsP}_{3}$ receptor predicts the presence of eight transmembrane regions (Mignery et al., 1990; De Camilli et al., 1990) although an alternative model with nine transmembrane regions has also been suggested (Furiuchi et al., 1989). Mutagenesis studies demonstrated that the receptor forms homotetramers by virtue of intersubunit interactions localized to the regions of the membrane spanning sequences, and that the ligand binding site is localized to the amino-terminal fourth of the receptor (Mignery and Südhof, 1990). Based on these results, a domain model of the receptor was suggested whereby the receptor contains an amino-terminal binding domain, a carboxy-terminal $\mathrm{Ca}^{2+}$ channel domain, and an intervening coupling domain that regulates the relationship between Ins $\mathrm{P}_{3}$ binding and $\mathrm{Ca}^{2+}$ channel gating. Consistent with this model, the phosphorylation sites of the $\mathrm{InsP}_{3}$ receptor were localized to the putative coupling domain (Mignery et al., 1990; Ferris et al., 1991b).

Although the cerebellar InsP $\mathrm{P}_{3}$ receptor is expressed at low levels in virtually all tissues investigated (Mignery et al., 1990), several lines of evidence suggest that there may be more than one type of $\mathrm{InsP}_{3}$ receptor. Biochemical data demonstrated that $\mathrm{Ins}_{3}$ binding has different characteristics in different tissues and that $\mathrm{Ca}^{2+}$ release by InsP $\mathrm{P}_{3}$ may be subject to different regulatory processes in different tissues (Guillemette et al., 1988; Palmer and Wakelam, 1989; Rossier et al., 1989; Ely et al., 1990; Pietri et al., 1990). These observations raise the possibility that different tissues may express different Ins $\mathrm{P}_{3}$ receptors. Furthermore, it has been suggested that the endoplasmic reticulum is subcompartmentalized with respect to its function as a $\mathrm{Ca}^{2+}$-storing organelle (Villa et al., 1991; Takei et al., 1992), indicating that different types of $\mathrm{InsP}_{3}$ receptors could be expressed in different subcompartments. For example, it is conceivable that a novel type of $\mathrm{InsP}_{3}$ receptor may be localized to peripheral elements of the endoplasmic reticulum and physically coupled to components of the plasma membrane, thereby mediating the observed plasma membrane $\mathrm{Ca}^{2+}$ flux (Irvine, 1990). Another possibility is that different $\mathrm{InsP}_{3}$ receptors are localized in different compartments of the endoplasmic reticulum, conferring different $\mathrm{Ca}^{2+}$ release properties on these compartments.

All of these possibilities imply the presence of additional types of InsP $\mathrm{P}_{3}$ receptors that are distinct from the only currently described $\mathrm{InsP}_{3}$ receptor. These receptors may nevertheless be structurally similar to this receptor although they differ from it in their intracellular targeting and/or 
regulation. We now report the presence and full length structure of a novel type of $\operatorname{InsP}_{3}$ receptor that fits these requirements. The presence of different types of Ins $\mathrm{P}_{3}$ receptors suggests that the intracellular $\mathrm{Ca}^{2+}$ signalling induced by Ins $\mathrm{P}_{3}$ may also be a function of the types and distributions of the InsP $\mathrm{P}_{3}$ receptors.

\section{Results}

In order to search for InsP $\mathrm{P}_{3}$ receptor related messages, a rat brain cDNA library was screened with an oligonucleotide corresponding to the last transmembrane region of the $\mathrm{InsP}_{3}$ receptor (Mignery et al., 1990). This region was chosen for screening because it constitutes the region of highest homology between the $\mathrm{InsP}_{3}$ and ryanodine receptors (Furiuchi et al., 1989; Mignery et al., 1989; Takeshima et al., 1989). In addition to multiple clones encoding the cerebellar $\mathrm{InsP}_{3}$ receptor, two overlapping clones were isolated that were different from the cerebellar $\mathrm{InsP}_{3}$ receptor clones (pI6 and pI15, Figure 1). Sequencing demonstrated that these clones encoded a novel transcript homologous to the $\mathrm{InsP}_{3}$ receptor. Oligonucleotides corresponding to the $5^{\prime}$ sequences of these and subsequent clones were then used to isolate further overlapping cDNA clones covering the entire coding region of the transcript and extending over $10.7 \mathrm{~kb}$ (Figure 1), and the sequences of all of these clones were determined.

The complete sequence of the $\mathrm{InsP}_{3}$ receptor related transcript was assembled from the sequences of the overlapping cDNA clones (Figure 2). Its translated amino acid sequence predicts synthesis of a protein containing 2701 amino acids with a total molecular weight of 307088 Daltons. The suggested initiation codon conforms well to the consensus sequence for initiator methionine codons (Kozak, 1989) and is preceded by an in-frame stop codon, suggesting that the sequence is full length with respect to the coding region. Clones containing poly(A) tails at two different positions in the $3^{\prime}$ untranslated region were isolated (Figure 1). Both poly(A) tails are preceded by AT-rich sequences that may serve as polyadenylation signals (underlined in Figure 2). Northern blots demonstrated the presence of two messages for this cDNA corresponding to approximately 9 and $11 \mathrm{~kb}$ in size (data not shown), suggesting that there is differential polyadenylation of the $3^{\prime}$ end of the message in vivo.

The amino acid sequence of the new protein was compared with that of the rat cerebellar InsP $\mathrm{P}_{3}$ receptor, revealing a high degree of homology between the two proteins with an overall sequence identity of $69 \%$. Alignment of the two sequences with each other (Figure 3) demonstrated that their homology extends over their entire length but shows a patchy distribution, with regions of identity separated by completely dissimilar sequence stretches. For example, hydrophobicity plots of both sequences suggested the presence of eight transmembrane regions (Mignery et al., 1990a and data not shown) which are underlined in Figure 3 and labeled M1 to M8. Most of the putative transmembrane regions are highly conserved but two transmembrane regions, M2 and M3, show very little sequence similarity. In addition, many but not all loops connecting transmembrane regions are poorly conserved, for example the sequence separating the sixth and seventh transmembrane regions contains no similarity except for two conserved cysteine residues whereas

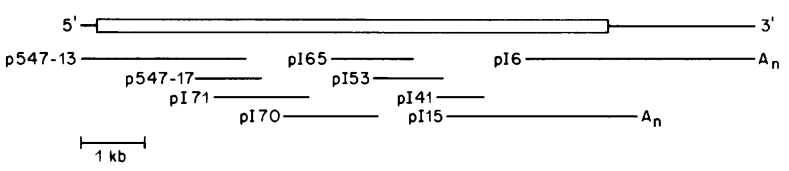

Fig. 1. Structure of the mRNA of the type $2 \mathrm{InsP}_{3}$ receptor (top) and distribution of the isolated cDNA clones (bottom). The open bar indicates the localization of the coding region in the mRNA. The scale of the graph is depicted in the lower left corner.

the transmembrane regions themselves are more than $90 \%$ identical. Similar patches of identical sequences separated by completely dissimilar regions can also be observed in other parts of the structures. In addition, deletions of one sequence relative to the other are observed, particularly in the coupling domain of the InsP $\mathrm{P}_{3}$ receptor that separates the transmembrane regions from the ligand binding domain. Interestingly, one of these deletions corresponds to an alternatively spliced region in the cerebellar $\mathrm{InsP}_{3}$ receptor (Ferris et al., 1991a), suggesting that the novel receptor may also be alternatively spliced.

Figure 3 also contains the partial sequence of a third mRNA that is related to the $\mathrm{InsP}_{3}$ receptor and was isolated by the polymerase chain reaction from a human kidney cDNA library (C.L.Newton, G.A.Mignery and T.C.Südhof, in preparation). This sequence shows the same pattern of similarity and diversity as described above, suggesting that there is a family of related sequences with a similar core of conserved residues.

The strong similarity between the novel sequence described here and the cerebellar Ins $\mathrm{P}_{3}$ receptor suggests that the new protein may represent a new type of $\operatorname{InsP}_{3}$ receptor. To test this hypothesis, we took advantage of the fact that we had previously localized the ligand binding domain of the cerebellar $\mathrm{InsP}_{3}$ receptor to the aminoterminal fourth of the receptor (Mignery and Südhof, 1990). Assuming that the ligand binding site of the putative new receptor would have a similar localization, we expressed the first 1078 residues of the new sequence as a soluble protein by transient transfection in COS cells (Figure 4). The ligand binding properties of the amino-terminal fragment of the novel receptor were compared to those of the corresponding homologous fragment from the previously characterized cerebellar InsP $\mathrm{P}_{3}$ receptor. In order to allow recognition of the two different recombinant proteins from the two receptors, the carboxy-termini of both proteins were fused to a 12 residue peptide epitope from the carboxy-terminus of the $116 \mathrm{~K}$ subunit of the vacuolar proton pump (Mignery and Südhof, 1990; Perin et al., 1991). After transient transfection, both proteins were expressed at high levels in soluble form in COS cells (Figure 4). Although the calculated molecular weights of the two proteins are very similar, their apparent mobility on SDS-gels differed slightly, possibly reflecting differences in their tertiary structure.

The InsP $\mathrm{P}_{3}$ binding properties of the recombinant proteins were then investigated in the cytosols of COS cells transfected with the expression constructs or control DNAs. Both recombinant proteins bound InsP $\mathrm{P}_{3}$ specifically, with the recombinant protein from the novel receptor having a slightly higher affinity than that of the corresponding fragment of the cerebellar $\mathrm{InsP}_{3}$ receptor (Figure 5; apparent $K_{\mathrm{d}} \mathrm{s}$ were $27 \mathrm{nM}$ and $89.5 \mathrm{nM}$, respectively). Furthermore, in spite of the considerable sequence 
differences between the two receptors, $\operatorname{InsP}_{3}$ binding to the recombinant proteins was displaced by different inositol phosphates to similar extents (Table I). In addition, $\mathrm{InsP}_{3}$ binding was very sensitive to heparin in both proteins. These results demonstrate that the protein described here represents a novel $\mathrm{InsP}_{3}$ receptor, from now on referred to as type 2 Ins $_{3}$ receptor as opposed to the cerebellar type $1 \mathrm{InsP}_{3}$ receptor. In spite of their sequence differences, both receptors have similar binding specificities although different affinities.

GGGACGCAGAGGGAGCGCGGGCCAGGGAGGAGGAGCGAAGGTGTAGGACAGAACTTCGCCAGGAACAGGAAACCCACGGCCGGGCAGGGGCGGCGACGGCGGCGCGGGGCCATC GCAGTGTCCCCAGCGGTGGCCTCGCTGCCGCGTCCCGAGCTGAGCCGCTTGGACCCCTCGGACTCAGAGGACCCAAGCTCACCCTTGCGAGCAGCGGAGACAGCGGGGACGGCC TCACGAGCGTGAAGCAGCATGTCTGACAAATGTCCAGCTTCCTCTACATTGGGGACATCGTGTCCCTGTACGCGGAGGGCTCGGTCAATGGCTTCATCAGCACCCTGGGGTTG $M$ S D $K$ M $M$ S S S F L Y I G D I V S L Y A E G S V N G F I S T L $L$ GTGGATGACCGTIGTGTGGTGCACCCGGAGGCGGGGGATCTTACCAACCCACCCAAGAAGTTCAGAGACTGCCTCTTCAAGGTGTGCCCCATGAATCGATACTCTGCCCAGAAG

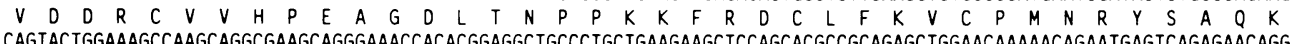
CAGTACTGGAAAGCCAAGCAGGCGAAGCAGGGAAACCACACGGAGGCTGCCCTGCTGAAGAAGCTCCAGCACGCCGCAGAGCTGGAACAAAAACAGAATGAGTCAGAGAACAGG $\begin{array}{llllllllllllllllllllllllllllllllllllll}Q & Y & W & K & A & K & Q & A & K & Q & G & N & H & T & E & A & A & L & L & K & K & L & Q & H & A & A & E & L & E & 0 & K & 0 & N & E & S & E & N & R\end{array}$ AAACTTTTGGGAGAAATTGTGAAATACAGCAAAGTTATACAACTACTGCACATAAAAAGCAACAAGTACCTCACCGTGACAAGAGGTTACCTGCCTTACTGGAGAAGAATGCC

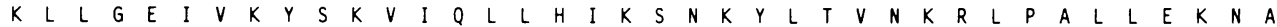
ATGCGTGTGTCCCTGGATGCTGCAGGGAACGAAGGTTCCTGGTTCTACATCCATCCCTTCTGGAAGCTGAGAAGCGAGGGTGATAATATCGTCGTGGGAGATAAAGTCGTTCTG

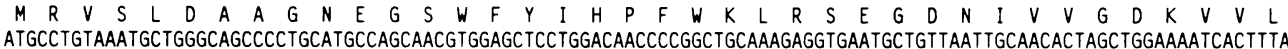

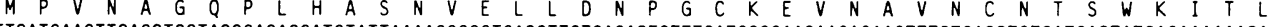
ITCATGAAGTTCAGCTCCTACCGAGAGGATGTATTAAAAGGGGGTGACGTTGTGAGACTGTTTCATGCGGAACAAGAGAAGTTTCTGACCTGTGATGACTATGAGAAAAAACAG

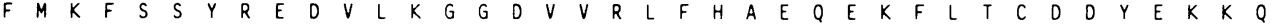
CACATTITCCTGCGGACGACCTTGCGTCAATCAGCAACGTCGGCCACTAGCTCTAAAGCACTCTGGGAGATAGAGGTGGITCACCATGATCCATGCCGCGGAGGTGCAGGACAG 1140

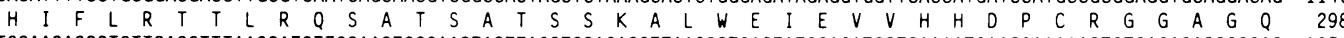
TGGAACAGCCTGTTCAGGTTTAAGCATCTTGCAACTGGGAACTACTTAGCTGCAGAGCTTAACCCTGACTATCGAGAiGCTCAAAATGAAGGAAAAACTGTGAGAGACGGGGAG 1254

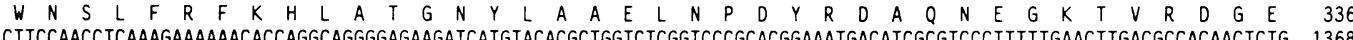
$L$ L $P$ T $S S$ K $K$ K $K$ K CAGAGAGCTGACTGCCTGGTTCCGAGGAACTCCTACGTGCGGTTGAGGCACCTGTGTACCAACACGTGGGTAACAAGCACCAGCATCCCCATAGACACGGAGGAAGAGAGGCCT 1482 $\begin{array}{llllllllllllllllllllllllllllllllllllllll}Q & R & A & D & C & L & V & P & R & N & S & Y & V & R & L & R & H & L & C & T & N & T & W & V & T & S & T & S & I & P & I & D & T & E & E & E & R & P & 412\end{array}$ GTCATGCTGAAAATTGGGACCTGCCAGACCAAGGAAGACAAAGAAGCCTTTGCCATCGTGTGCGTCCCGCTGTCTGAGGTCCGAGACCTGGACTTTGCCAACGATGCCAACAAA 1596 $\begin{array}{llllllllllllllllllllllllllllllllllllll}V & M & L & K & I & G & T & C & Q & T & K & E & D & K & E & A & F & A & I & V & C & V & P & L & S & E & V & R & D & L & D & F & A & N & D & A & N & K\end{array}$ GTGTTGGCCACCACGGTGAAGAAGCTGGAGAACGGCAGCATCACCCAGAATGAGAGGAGGTITGTGACTAAGITGITGGAAGACCTIATITICTITGTGGCTGATGTGACCAAC 1710

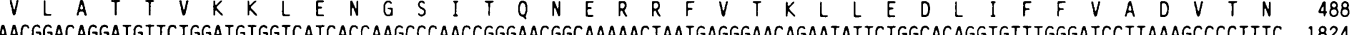

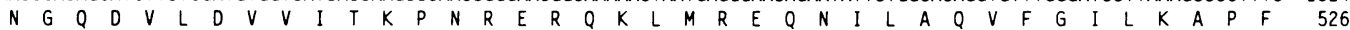
AAGGAGAAGGCTGGGGAAGGTTGATGCTGAGGCTGGAGGACCTGGGCGACCAGCGCTATGCCCCGTACAAGTACGTGCTGCGTCTCTGCTACCGTGTGCTGAGGCATTCGCAG 1938

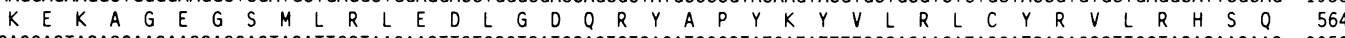
CAGGACTACAGGAAGAACCAGGAGTACATTGCTAAGAACTTCTGCGTCATGCAGTCTCAGATCGGCTATGATATTTTGGCAGAAGATACGATCACAGCCTTGCTACACAACAAC 2052 $\begin{array}{cccccccccccccccccccccccccccccccccccccccccc}Q & D & Y & R & K & N & Q & E & Y & I & A & K & N & F & C & V & M & Q & S & Q & I & G & Y & D & I & L & A & E & D & T & I & T & A & L & L & H & N & 602 \\ \text { CGCAAACTCCTGGAGAAACACATCACGGCCAAAGAAATCGAGACCTTCGTCAGCCTGCTCAGGAGGAACCGGGAGCCGAGGTITCTGGACTATCTGTCTGACCTCTGCGTGTCC } & 2166\end{array}$

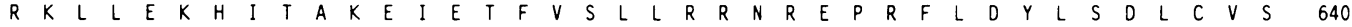
AATAGCACCGCCATCCCTGTGACTCAGGAGCTCATCTGCAAATTCATGCTGAGCCCCGGCAATGCGGACATCCTCATTCAGACGAAGCTGGTGTCCATGCAAGTGGAAAACCCC 2280 $N$ N S $T$ T ATGGAGAGCTCCATCCTTCCCGATGACATCGACGACGAGGAGGTTTGGCTTTACTGGATTGACAGCAACAAGGAGCCTCACGGCAAGGCCATCAGGCACCTGGCCCAGGAGGCC 2394 $M$
AGGGAAGGCACCAAGGCTGACCTAGAAGTCCTGACCTATTACAGGTACCAGCTAAACCTCTTTGCAAGGATGTGCTTGGACCGCCAGTACCTGGCCATCAACCAGATTTCAACA $\begin{array}{llllllllllllllllllllllllllllllllllllllll}R & E & G & T & K & A & D & L & E & V & L & T & Y & Y & R & Y & Q & L & N & L & F & A & R & M & C & L & D & R & Q & Y & L & A & I & N & 0 & I & S & T & 754\end{array}$ CAGCTGTCTGTAGACCTGATCCTGCGGTGCGTGTCTGACGAGAGCCTGCCCTTIGACCTCCGAGCCTCTTTCTGCCGACTCATGCTCCACATGCATGTGGACCGGGACCCCCAG 2622 $\begin{array}{llllllllllllllllllllllllllllllllllllllll}Q & L & S & V & D & L & I & L & R & C & V & S & D & E & S & L & P & F & D & L & R & A & S & F & C & R & L & M & L & H & M & H & V & D & R & D & P & 0 & 792\end{array}$ GAGTCTGTGGTGCCTGTCCGCTACGCCCGGCTCTGGACTGAGATCCCCACCAAGATCACGATCCATGAGTATGACTCCATCACAGACTCTTCCAGAAATGACATGAAGAGGAAG 2736 $\begin{array}{llllllllllllllllllllllllllllllllllllllllllll}E & S & V & V & P & V & R & Y & A & R & L & W & T & E & I & P & T & K & I & T & I & H & E & Y & D & S & I & T & D & S & S & R & N & D & M & K & R & K & 830\end{array}$ TITGCCCTGACAATGGAATTTGTCGAAGAATATTTGAAAGAAGTTGTGAATCAACCGTTTCCTTITGGGGACAAAGAGAAAAATAAACTGACATTTGAGGTGGTCCACCTGGCC 2850

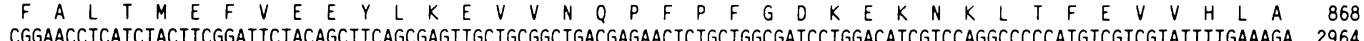
F Y E L L R L T R T L L A I L D I V O A P M S S Y F E R 906 ITAAGCAAGTTICAAGATGGAAGCAACAATGTCATGAGGACCATCCACGGGTGGGAGAGATGATGACACAGATGGTGCTCAGCAGAGGCTCCATTTTCCCAGTGAGCTGGCCC 3078 $\begin{array}{llllllllllllllllllllllllllllllllllllllll}L & S & K & F & Q & D & G & S & N & N & V & M & R & T & I & H & G & V & G & E & M & M & T & Q & M & V & L & S & R & G & S & I & F & P & V & S & W & P & 944\end{array}$ GACGCACAGCCCAGCGTCCACCCCAGCAAGCAGGCGAGCCCCGGCGAGCAGGAGGATGTGACGGTTATGGATACCAAGCTAAAGGTTATCGAGATTTTACAGTTCATCCTGAGT 3192 $\begin{array}{llllllllllllllllllllllllllllllllllllllll}D & A & Q & P & S & V & H & P & S & K & Q & A & S & P & G & E & Q & E & D & V & T & V & M & D & T & K & L & K & V & I & E & I & L & Q & F & I & L & S & 982\end{array}$ GICAGGCTGGACTACCGTATCTCTTACATGCTGTCCATATACAAGAAGGAATITGGGGAGAATGATGGCAACGGTGACCCCTCTGCCAGCGGCACCCCGGAAACATTGCTACCA 3306

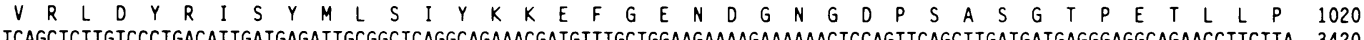

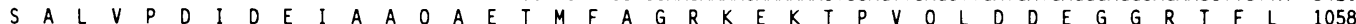
AGAGTCCTCATCCACCTGATCATGCATGACTATGCTCCACTGCTGTCCGGGGCCCTGCAGCTGCTGTTCAAACACTTCAGCCAGAGAGCAGAAGTGCTTCAGGCCTTCAAACAG 3534

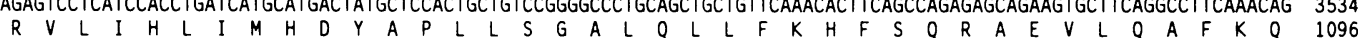
$\begin{array}{lllllllllllllllllllllll}R & V \\ \text { GTGCAGTTACTGGTGTCGAACCAAGATGTCGATAACTACAAGCAAATCAAGGCAGACCTGGACCAACTTCGGCTGACGGTGGAGAAGTCCGAGCTATGGGTGGAAAAGAGCGGC } & 3648\end{array}$ $\begin{array}{lllllllllllllllllllllllllllllllllllllll}V & Q & L & L & V & S & N & Q & D & V & D & N & Y & K & Q & I & K & A & D & L & D & Q & L & R & L & T & V & E & K & S & E & L & W & V & E & K & S & G & 1134\end{array}$ AGCTATGAGAATGGAGACATGGGCGAGGGCCAGGCCAAAGGAGGCGAAGAGGCAAACGAGGAGTCCAACCTCTTGAGCCCGGTGCAGGACGGCGCGAAGACACCTCAGATCGAC 3762 $\begin{array}{lllllllllllllllllllllllllllllllllllllll}S & Y & E & N & G & D & M & G & E & G & Q & A & K & G & G & E & E & A & N & E & E & S & N & L & L & S & P & V & Q & D & G & A & K & T & P & 0 & I & D & 1172\end{array}$ AGCAACAAGGGCAACAACTACCGGATCGTCAAGGAGATCTTGATTAGGCTGAGTAAGCTCTGTGTGCAGAACAAGAAATGTCGTAATCAACACCAACGGTTGCTGAAAAATATG 3876 $\begin{array}{lllllllllllllllllllllllllllllllllllllllll}S & N & K & G & N & N & Y & R & I & V & K & E & I & L & I & R & L & S & K & L & C & V & O & N & K & K & C & R & N & Q & H & 0 & R & L & L & K & N & M & 1210\end{array}$ GGGGCTCACTCCGTGGTACTGGACCTTCTGCAGATCCCCTATGAGAAGACCGACGAGAAGATGAACGAGGTGATGGACTTAGCCCACACCTTCCTGCAGAACTTCTGCCGCGGG 3990 $\begin{array}{lllllllllllllllllllllllllllllllllllllll}G & A & H & S & V & V & L & D & L & L & Q & I & P & Y & E & K & T & D & E & K & M & N & E & V & M & D & L & A & H & T & F & L & Q & N & F & C & R & G & 1248\end{array}$ AATCCACAGAACCAAGTTCTCCTTCACAAGCATCTCAACCTGTTCCTAACCCCTGGACTCCTGGAGGCGGAAACCATGCGGCACATCTTCATGAACAATTACCATCTGTGCAAT 4104 GAGATCAGCGAGAGGGTGGTCCAGCACTTTGTGCACTGCATTGAGACACACGGCCGCCACGTGGAGTACCTGAGGTTCCTGCAGACCATCGTGAAAGCAGACGGCAAATACGTG 4218 E I I S E $R$ R V V V AAGAAATGCCAGGACATGGTGATGACCGAGCTGATCAATGGGGGTGAAGACGTACTGATATTCTACAACGACCGAGCCTCATITCCCATCCTTCTCAACATGATGTGCTCCGAG 4332

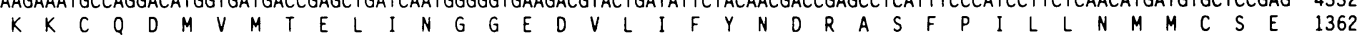
AGAGCCCGTGGGATGAGAGCGGCCCCCTGGCCTACCATATCACCCTTGTGGAGTTGCTGGCAGCGTGCACTGAGGGGAAGAACGTCTACACGGAGATCAAGTGCAATTCTCTC 4446 $\begin{array}{llllllllllllllllllllllllllllllllllllllll}R & A & R & G & D & E & S & G & P & L & A & Y & H & I & T & L & V & E & L & L & A & A & C & T & E & G & K & N & V & Y & T & E & I & K & C & N & S & L & 1400\end{array}$ TTGCCTCTGGACGACATAGTGAGGTGGTGACCCACGACGACTGCATTCCTGAGGTAAAAATTGCATACGTGAACTITGTCAACCATTGTTACGTTGACACAGAAGTGGAGATG 4560

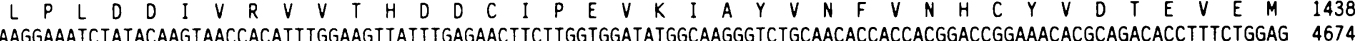
$K$ K I Y T S N H I W K L F E N F L V D M A R V C N T T T D R K K H A AGGTGTGTGACAGAGTCAGTCATGAACATCGTGAGCGGCTTCTTCAACTCGCCGITTTCGGATAACAGTACCAGCCTCCAGACCCATCAGCCGGTCTTTATCCAGCTGCTGCAG 4788

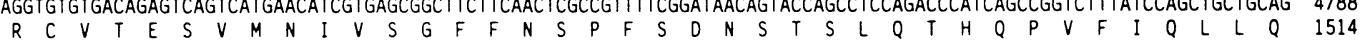

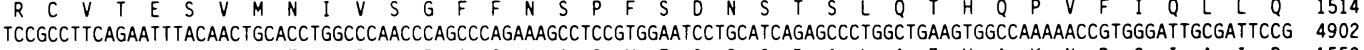
$\begin{array}{lllllllllllllllllllllllllllllllllllllll}S & A & F & R & I & Y & N & C & T & W & P & N & P & A & Q & K & A & S & V & E & S & C & I & R & A & L & A & E & V & A & K & N & R & G & I & A & I & P & 1552\end{array}$ GTGGATTTGGACAGCCAGGTCAACACACTCTTCATGAAGAATCACTCAAGTACAGTGCAGAGGGCGGCCATGGGCTGGAGGCTCTCTGCTCGCTCCGGACCTCGCTTCAAGGAA 5016 GCTCTTGGAGGGCCAGCATGGGACTACAGGAATATTATTGAAAAGTTACAGGATGTCGTGGCCTCTCTGGAACAGCAGTTCAGCCCCATGATGCAGGCTGAGTTCTCTGTGCTT 5130 
A L G G P A W D Y Y R N I I I E K L Q Q D V V V A S L L E Q Q GTGGATGTGCTGTACAGCCCGGAGCTACTGTTCCCAGAAGGAAGCGATGCCAGGATACGATGTGGTGCCTTCATGTCCAAGCTGATTAATCACACAAAGAAACTGATGGAAAAA 5244

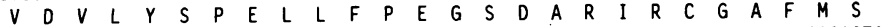
GAAGAAAAACTGTGCATTAAAATCCTCCAGACGTTACGGGAGATGCTGGAGAAGAAAGACAGCTTCATGGAAGAGAGTAGCACATTACGGAAAATCCTCCTGAATCGGTACTTC 5358

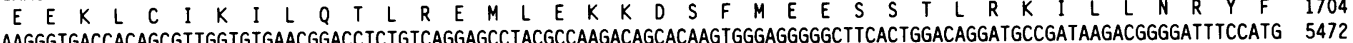
K G D H S V G V N G P L S G A Y A K T A Q V G G G F T G Q D A D K T G I S M 1742 TCCGATATCCAGTGTTIGCTGGACAAGGAAGGGGCCTCCGAACTTGTCATCGATGTCATTGTGAACACCAAAAACGACAGGATTTTTTCCGAGGGCATCTTGCTTGGCATTGCC 5586

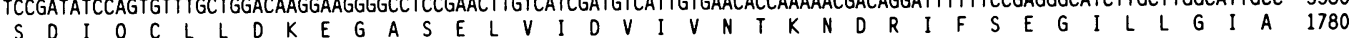
CTGCTGGAAGGGGAG $L L E G G N T O F$ S S F Y O O H E O K K S E K F F K V L Y D R M K A A Q 1818 作

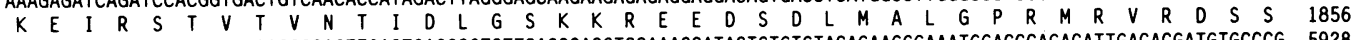
CTGCATTTGAAAGAGGGAATGAAAGGGCAGTTGACTGAGGCGTCTTCAGCCACGTCCAAAGCATACTGTGTGTACAGAAGGGAAATGGACCCAGACATTGACACGATGTGCCCG 5928 CTGCATTGAAAGAGGGAATAAAGGGAG T K F S S A T S K A Y C V Y R R E M D P D I D T M C P 1894 $L$ L $H$ K K E G M K G O L GGACAGGAAGCAGGAAGTGCGGAGGAAAAGTCTGCAGAGGAAGTTACCATGAGCCCAGCCATCACTATCATGCGGCCCATCCTCAGGTTCCTGCAGTTACTGTGTGAGAATCAC 6042

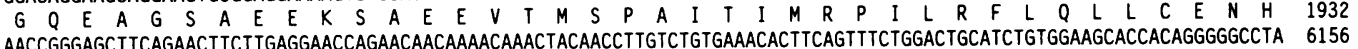
N R E L O N F L R N Q N N K T N Y N L V C E T L O F L D C I I C G S T T T G G L 1970 GGCCTGCTGGGGCTCTACATCAATGAGAAGAACGTAGCTCTGGTCAACCAGACCCTGGAGAGCTTGACCGAGTACTGCCAGGGCCCGTGTCATGAGAACCAGACCTGTATCGCC 6270 GCC L L G Y I N E K N V A L V N Q T L E S L T E Y C Q G P C H E N Q T C I A 2008 ACTCACGAATCCAATGGGATTGACATCATCATCGCCTTGATTCTGAGTGACATCAACCCTCTGGGCAAGTACAGAATGGACCTGGTGCTACAGCTGAAGAACAACGCCTCCAAG 6384 ACTCACGAATCCAATGGGATTGACATCATCATCGCCTTGATTCTGAGTGACATCAACCCTCTGGGCAAGTACAGAATGGACCTGGTGCTACAGCTGAAGACAACGCCTCCAAG 6384

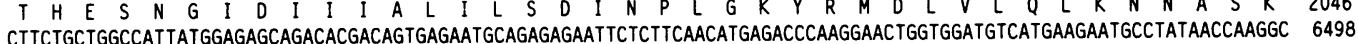
$L$
$L$ CTGAATGTACCATH CTA CAGCAGATGCTCAAGCCTGGATCCGATCCAGAGGAAGGGGATGAAGCCTTGAAGTACTATGCCAACCACACCGCACAGATCGAGATTGTGCGGCACGACCGGACTATGGAGCAG 6726 CAGCAGATGCTCAAGCCTGGATCCGATCCAGAGGAAGGGGATGAAGCCTTGAAGTACTATGCCAACCACACCGCACAGATCGAGATTGTGCGGCACGACCGGACTATGGAGCAG

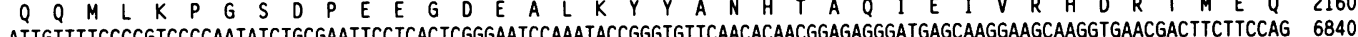
ATTGTTTTCCCCGTCCCCAATATCTGCGAATTCCTCACTCGGGAATCCAAATACCGGGTGTTCAACACAACGGAGAGGGATGAGCAAGGAAGCAAGGTGAACGACTTCTTCCAG 6840 \begin{tabular}{llllllllllllllllllllllllllllllllllllll}
$I$ & $V$ & $F$ & $P$ & $V$ & $P$ & $N$ & $I$ & $C$ & $E$ & $F$ & $L$ & $T$ & $R$ & $E$ & $S$ & $K$ & $Y$ & $R$ & $V$ & $F$ & $N$ & $T$ & $T$ & $E$ & $R$ & $D$ & $E$ & $Q$ & $G$ & $S$ & $K$ & $V$ & $N$ & $D$ & $F$ & $F$ & $Q$ \\
\hline
\end{tabular} O T E D L Y N E M K W O K K I R N N P A L F W F S R H I S L W G S I S F N L 2236 GCTGTGTTCATCAACCTGGCCGTGGCTCTCTTCTACCCATTCGGGGATGACGGCGATGAAGGTACGCTCTCCCCGCTGTTCTCAGCCCTCCTTTGGGTAGCAGTGGCGATCTGC 7068 A V F I N L A V A L F Y P F G D D G D E G T L S P L F S A L L W V A V A I C 2274 ACGTCTATGCTGTTCTTCTTCTCCAAGCCTGTGGGCATCCGGCCATTCCTTGTTITATCATGCTAGATCAATATACACCATCGGTCTGGGGCAACGCTAATACTTCTTGT 7182 T S M L F F F S K P P V G I R P F L V S I M L R S I Y T I G L G P T L I $L$ GCTGCCAATCTATGCAATAAAATCGTGTTCCTGGTGAGTITTGTGGGAAACCGAGGCACATTCACCCGAGGGTACCGAGCAGTCATTCTGGACATGGCCTTTCTTTACCACGTG 7296

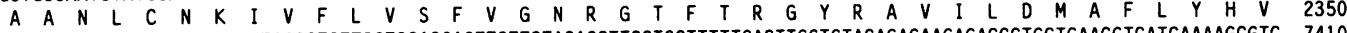
GCCTATGTCTTGGTTTGCATGCTTGGCCTCTTCGTCCACGAGTTCTTCTACAGCTTCCTGCTTTTTGACTTGGTGTACAGAGAAGAGACCCTGCTGAACGTCATCAAAAGCGTC 7410 $A$ Y V L Y C M L G L F V H E F F Y S F L L F D L V Y R E E T L L N V I K S V 2388 ACACGGAATGGCCGCTCCATCATCCTGACTGCGGTCTTGGCTCTTATCCTGGTCTACCTGTTCTCCATCATCGGCTTCCTTITCTTAAAGGATGACTTCACCATGGAGGTGGAC 7524

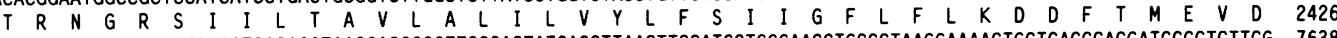
AGATTGAAAAACAGAACTCCAGTCACAGGTAACGACGGGGTTCCCACTATGACCTTAACTTCCATGCTGGGAACCTGCCCTAAGGAAAACTGCTCACCCACGATCCCCTCTTCG 7638

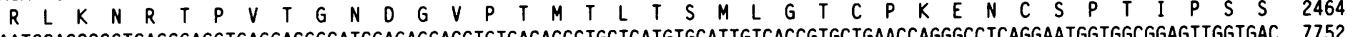
AATGCAGCCGGTGAGGGAGGTGAGGACGGCATCGAGAGGACCTGTGACACCCTGCTCA V T V L L N Q G G L R R N G G G V G G GTGCTGAGACGACCCTCGAAGGATGAGCCTTTGTTTGCTGCCCGGGTGGTCTACGACCTCCTTTTCTTCTTCATCGTCATCATCATCGTCCTTAACCTGATTTTTGGTGTATC 7866 ATTGACACTTICGCTGACCTCAGGAGTGAGAAGCAGAAGAAAGAAAAAATTCTCAAGACAACCTGCTTCATCTGTGGCTTGGAGAGGGACAAGTTTGATAACAAGACGGTGTCC 7980 I D T F A D D L R S E K $K$ Q K K K E K I I L K T T C F I C G L E R D K F D N K T V S 2578 TITGAGGAGCACATCAAGTCAGAGCACAACATGTGGCATTACTTGTACTTCATCGTCCTGGTGAAGGTGAGGACCCAACAGAATACACAGGGCCTGAGAGCTACGTGGCTCAG 8094

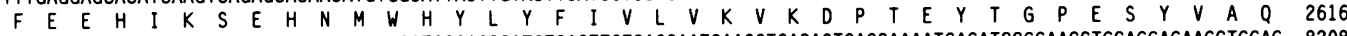
ATGATCACAGAGAAGAATTTAGACTGGTTTCCTCGGATGCGAGCCATGTCACTTGTCAGCAATGAAGGTGACAGTGAGCAAAATGAGATCCGGAACCTGCAGGAGAAGCTGGAG 8208 $M$ I T E K N L D W F P R M R A M S L V S N E G D S E Q N N E I R N L Q E K L E 2654 TCCACTATGAGTCTGGTGAAACAGTTGTCGGGGCAGCTGGCAGAGCTGAAGGAGCAGATGACAGAACAAAGGAAAAACAAGCAAAGGCTGGGCTTCCTTGGATCAAACACACCC 8322

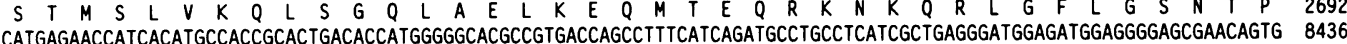
H E N H H M P P H GCTATTGTTGAAAAGCTGAAAACAACCAAGTGCCAAGGTGCTGAGCCATTCAGCTCCCAGAACAATCTGTAACTGGGTTGCACGCT GAGAGGG TCAAGCTIGGAAAAAAAC 8550 AAAACAAAACAGTATAGGGCACAGCCTCTCCATGTGGCAGGAAGCGGCCAGCCTGAGGGGCTGGAGGGGGGGGAGAGTCTGATCGGGGGGGCAGCCTCCCTCCTCGA 8664 CGATAGGCCTTGGACTTGTCTCACACACTGACTGCAGTGTCCATCGTGGCTGGTTGAAATTITTTCTTCAAACTGTGGCACTGGGGTAGCGAGACAGGAAGCCACACTCTGCT 8778 (1)

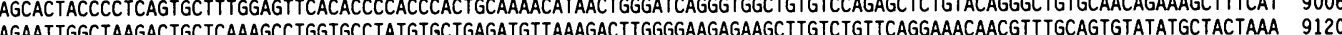
AGAATTGGCTAAGACTGCTCAAAGCCTGGTGCCTA TGGCCAACACTGAGAAGCGTTACTCGGGTGGCCTTGCCTITAAAAGCAGGTTAATTTATATTTGAGGTGCTTGACCCAA 9234 GTATGTGGGCATTGAAGGAAAAACTCGAGGGTAACAGCCCAACACTGAGAAGCGTTACTCGGGTGGCCTTGCCTTTAAAAGCAGGTTAATTTATATTTGAGGTGCTTGACCCAA 9234 GTGCTTGCGATCCCCGAAGCTGAGGGGGTTGGGGGAATCTAAGAATTGCATTGACTACGTAAGAATATGCAACATTTCAACAGCACATTTAAGGCTGITAGAGTAACAGCTIT 9348 TCAAAATGTAGGCAGATCATAATTCCTTCCCCCTAACTGTTTTCATGITGACTCATGCAACCAGATTTTGTCAAGGGAAAAAATAACTTGGAGGGGAGATTCCTTTTGGAGAC 9462 CTITAAACTCTCAGCCACCAAGGGCCACATGGGGTTTGTTACAGCAAGTTITAAAGCTTTCTGGCATTTCAATACAGTTTTAGCAGTTGGCTCCTAAAAATAAACAAACAAACA 9690 AACAAGTCAAATCCTGGGTGGTATAGACCCACTGTACAGGCAGAAACTCAAATGGCTTACCTTCTCTTGGGTGTAGCCTTGGAACCACCGAGATGTACTTTCTAACTAAATGC 9804 TGCACACACTAGGACCACCCAGAGITACAAAGTGCTCTTGGACAGGGCCCTGCTCCCCTTAGCCTAAAGTGGAAAGATGCTGGTGCCTTGACCTCAGGTGTTCTAGGCTAAAAC 9918 ACTGCACCACTGACCCTCGACTCTTGTAAGAGACCTGTCTTCAGTTACCCTTAATTCTTTGCTAAAGCTTCAAACTATAATCACAAACATTTTTTTGAAGGGTATCACCAAAAT 10032 TAGGCCACGGCAGCCATTTTTAAAGCTCAGGACCATGGATACTTCGACGGTATTTGGTAAAAGTAAAATAGGCTGGGCGATCAGAAGGATAGGAGCTGCTTCAGTCTGCTGTAA 10146 CCTAACCCTCCATCCGTTGTGCCAGCAGCCCACAGTTCGACACCGTGGCTTCCTCCTGTGGGAACTATCCAAAAGATTTCACCTAACGAGACTCTCACTATGTCAAAATTAATT 10260 TITITCAGTTGTACTTITTCITICCCCTCTACAAGATGAAAGATIATAAAGTGAAATTITATAAACATCAACCAAAGCAAAGGCACTTICTAGGACACTGACTTCATAAAGATG 10374 ITAGAAATGAGGAAATTIGATGTCAGGTGAGAGTTTATACTTGTTATGTATGGTTACTAATGCAGCGGGTGTAGCTGACTGAAGTTTGATATAGTTGTATTCTCACATITATAG 10488 TGATATTAAAAATACAGTCACCTCTGACTTCTATGCCAGGGAAGGCTITTTTAAATITTAGGTTATTAAAATCCATATTGGTTAAAAGTCACAGTTCCCACTGAATAATATTTT 10602 TAATTCACAGAAGgGTTTGTTCCCAGGTGTGCTCCATTCTGCATCACAAGAGTITAGTCGAAGACATTITCTAAATTAAAATAAITGTTTGTAATíaGAAAAAAAA

10708

Fig. 2. Nucleotide and translated amino acid sequences of the type $2 \mathrm{InsP}_{3}$ receptor from rat. The sequence was assembled from the sequences of the cDNA clones shown in Figure 1. The deduced amino acid sequence is shown in single letter code below the nucleotide sequence, and both sequences are numbered on the right. The in-frame stop codon in the $5^{\prime}$ untranslated region preceding the initiator codon is underlined. The position of the poly(A) tail in pI15 is shown by an asterisk, and sequences that might serve as polyadenylation signals for this poly(A) tail and for the one at the end of clone pI6 are underlined. The following sequence differences were noted between different cDNA clones: The C at position 2289 was a $\mathrm{T}$ in p547-17 (silent change); the $\mathrm{G}$ at position 2311 was a $\mathrm{C}$ in pI71, changing $\mathrm{D}$ at position 689 to $\mathrm{H}$; the $\mathrm{G}$ at position 2397 was a $\mathrm{T}$ in pI71 (silent change); the $T$ at position 2604 was a $C$ in p547-17 (silent change); the $G$ at position 3283 was a $T$ in pI 70 , changing $G$ at position 1013 to $C$; the $\mathrm{G}$ at position 3473 was a $\mathrm{C}$ in pI70, changing $\mathrm{G}$ at position 1065 to $\mathrm{A}$; the $\mathrm{T}$ at position 4013 was a $\mathrm{C}$ in pI70, changing $\mathrm{L}$ at position 1256 to $\mathrm{P}$; the $C$ at position 4770 was a $T$ in pI65 (silent change); the $G$ at position 4890 was an $A$ in pl65 (silent change); the $G$ at position 4911 was an $A$ in pl65 (silent change); the $\mathrm{G}$ at position 5929 was an A in pI15 (silent change); the $\mathrm{G}$ at position 7396 was a A in pI15, changing V at position 2384 to $\mathrm{I}$; and the $\mathrm{A}$ at position 8327 was a $\mathrm{T}$ in pI6, changing $\mathrm{E}$ at position 2694 to $\mathrm{V}$. In addition, clone pI70 had an out-of-frame deletion from nucleotide 3311 to 3378 . These sequence data are available from the EMBL/GenBank/DDBJ databases under accession number X61677 ITPR2. 


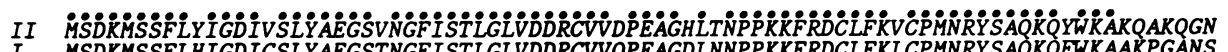
I MSDKMSSFLHIGDICSLYAEGSTNGFISTLGLVDDRCVVQPEAGDLNNPPKKFRDCLFKLCPMNRYSAQKQFWKAAKPGANS

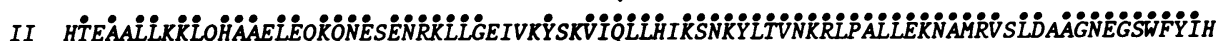

I TTDAVLLNKLHHAADLEKKQNETENRKLLGTVIQYGNVIQLLHLKSNKYLTVNKRLPALLEKNAMRVTLDEAGNEGSWFYIQ

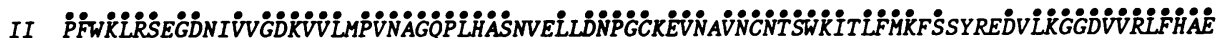

I PFYKLRSIGDSVVIGDKVVLNPVNAGQPLHASSHQLVDNPGCNEVNSVCNT SWKIVLFMKWSDNKDDILKGGDVVRLFHAE

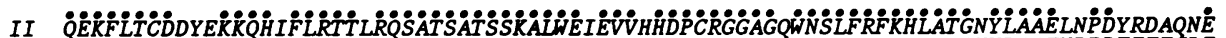

I QEKFLTCDEHRKX OHVFLRTTGRQSAT SAT SSKALWEVEVVQHDPCRGGAGYWN LFRFKHLATGHYLAAEVDPDFEEECLE

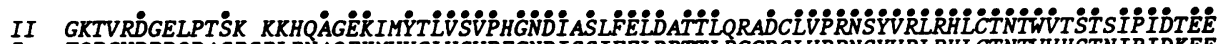

I FQPSVDPDQDASRSRLRNAQEKMVYSLVSVPEGNDISSIFELDPTTLRGGDSLVPRNSYVRLRHLCTNTVHSTNIPIDKEE

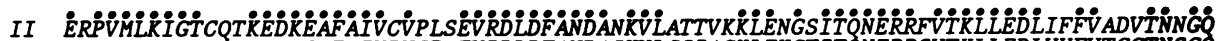

I EKPVMLKIGTSPLKEDKEAFAIVPVSPAEVRDLDFANDASKVLGSI AGKLEKGTITQNERRSVTKLLEDLVYFVTGGTNSGQ

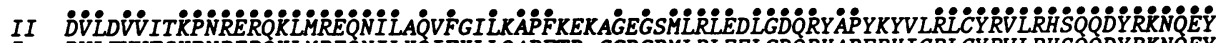

I DVLEVVFSKPNRERQKLMREQNILKQIFKLLQAPFTD CGDGPMLRLEELGDQRHAPFRHICRLCYRVLRHSQQDYRKNQEY

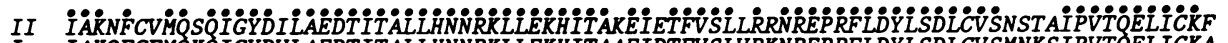

I I AKQFGFM $Q$ K IGYDVLAEDT ITALLHNNRKLLEKHITAAE IDTFVSLVRKNREPRFLDYLSDLCVSMNKSIPVTQELICKA

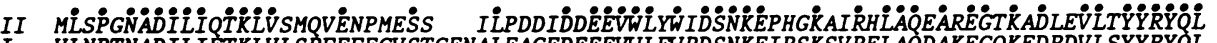

I VLNPTNADILIETKLVLSRFEFEGVSTGENALEAGEDEEEVWLFWRDSNKEIRSKSVRELAQDAKEGQKEDRDVLSYYRYQL

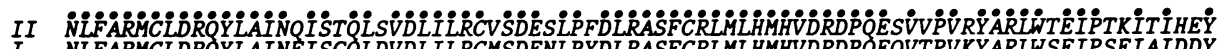

I NLFARMCLDRQYLAINEISGQLDVDILRCMSDENLPYDLRASFCRLMLHMHVRDPQEVTPVKYARLWEETSEIAIDDY

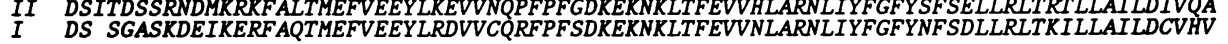

II PMSSYFERLSKFOD G̊SNNVํํRTI

II PMSSYFERLSKFQD GSNNVMRTIHGVGMMTQMVL RGGGFLPMTPMAAAPEGNVKQAEP EKEDIMVMDTKLKIIEILO

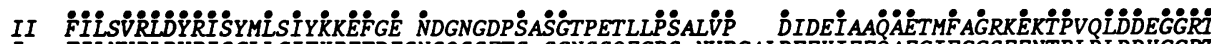

I FILNVRLDYRISCLLCIFKREFDESNSQSSETS SGNSSQEGPS NVPGALDFEHIEEQAEGIFGGSEENTPLDLDDHGGRT

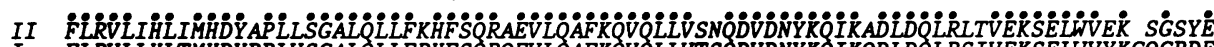

I FLRVLLHLTMHDYPPLV SGAL QLLFRHF QRQEVLQAFKQVQLLVTS QDVDNYK IKQDLDQLRSIVEKSELWVYKGQGPDE

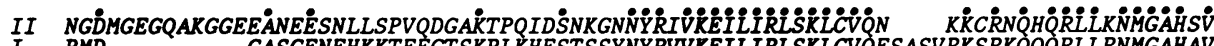

I PMD GASGENEHKKTEEGTSKPLKHESTSSYNYRVVREILIRLSKLCVQESASVRKSRKQQQRLLRNMGAHAV

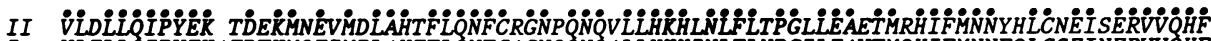

I VLELLQIPYEKAEDTKMQEIMRLAHEFLQNFCAGNQQNQALLHKH INLFL NPGILEAVTMQH IFMNNFQL C SE INERVVQHF

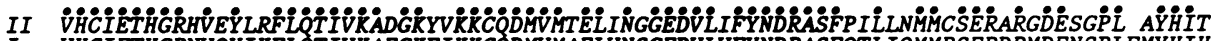

I VHCIETHGRNVYIKFLQTIVKAEGKFIKKCQDHVMAELVNSGEDVLVFYNDRASFQTLIQMMRSERDRMDENSPLFMYHIH

II

I LVELLAVCTEGKNVYTEIKCNSLLPLDDIVRVVTHEDCIPEVKIAY INFLNHCYVDTEVEMKEIYT SNHMWKLFENFLVDIC

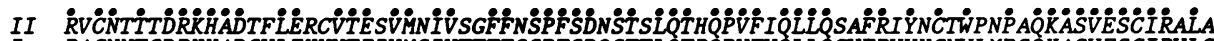

I RACNNTSDRKHADSVLEKYVTEIVMSIVTTFF SSPFSDQSTTLQTRQPVFV QLLQGVFR YHCNWLMP SQKASVESCIRVLS

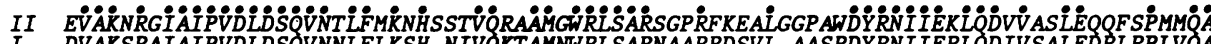

I DVAKSRAIAIPVDLDSQVNNLFLKSH NIVQKTAMNWRLSARNAARRDSVL AASRDYRNI IERLQDIVSALEDRLRPLVQA

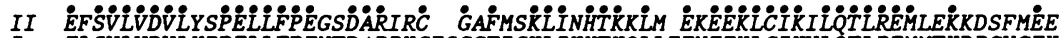

I ELSVLVDVLHRPELLFPENTDARRKCESGGFICKLIKHTKQLLEENEEKLCIKVLQTLREMMTKDRGYGEKQISIDELENAE

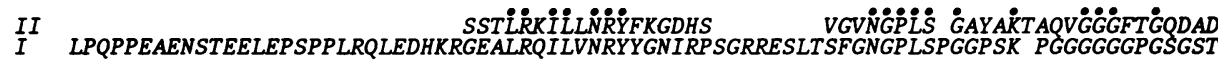

82

164

246

328

409

410

491

573

655
655

734
737

816

898
900

978

979

1056

1137

1141

1215

1296

1295

1377
1377

1459

1459

1541

1541

$\frac{1623}{1621}$

1691

1703

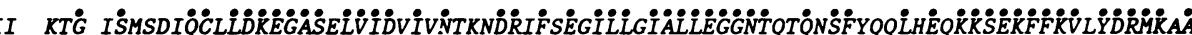

I SRGEMSLAEVQCHLDKEGASNLVIDLIMNASSDRVFHESILLAIALLEGGNTTIQHSFFCRLTEDKKSEKFFKVFYDRMKVA

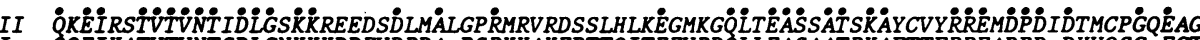

I QQEIKATVTVNTSDLGNKKRDDEVDRDA PSRKKAKEPTTQITEEVRDQLLEASAATRKAFTTFRREADPD DHYQSG EGT

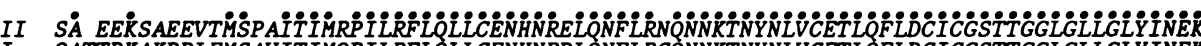

I QATTDKAKDDLEMSAVITIMQP ILRFLQLLCENHNRDLQNFLRCQNNKTNYNLVCETLQFLDCICGSTT GGLGLLGLYINEK

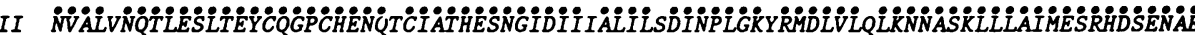

I NVALINQTLESLTEYCQGPCHENQNCI ATHESNGIDI ITALI LND INP LGKKRMDLVLELKNNASKLLLAIMESRHD SENAE

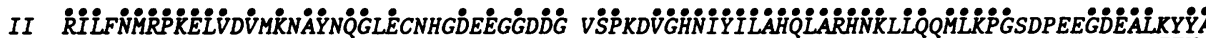

I RILYNMRP KELVEVIKKAYMQG EVEFEDGENGEDGAASPRNVGHNIYILAHQLARHNKELQTMLKPG GQVDGDEALEFYA

II NHOOAOQOOOEIVOR

I KHTAQIEIVRLDRTMEQIVFPVPSICEFLTKESKLRIYYTTERDEQGSKINDFFLRSEDLFNEMNWQKKLRAQPVLYWCARN

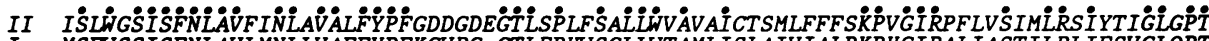

I MSFWSSISFNLAVLMNLLVAFFYPFKGVRG GTLEPHSGLLWTAMLISLAIVIALPKPHGIRALIASTILRLIFSVGLOPT

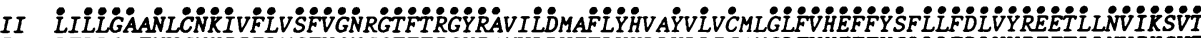
I
III LFLLGAENVCNKIIFLMSFV GNGTETRGYRAMVLDVEFLYHLLYLLICAMGLFVHEFFYSLLLFDLVYREETLLNVIKSVT
$M 4$

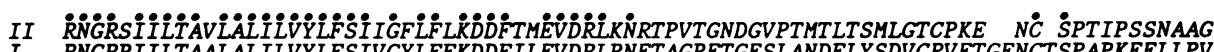

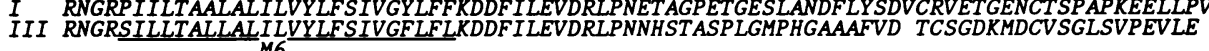

1736
1784

1817

1866

1899

1980

2027

2062

2143

2225

2307

2352

2389

2434

2468

2516

II EGGGEGI

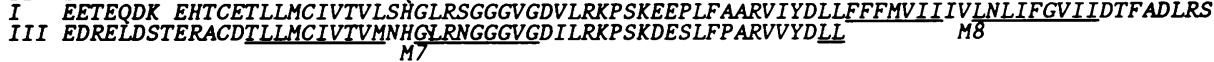

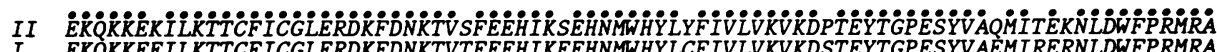

I EKQKKEE ILKTTCF ICGLERDKF DNKTVTFEEH I KEEHNYWHYLCF IVLVKVKDSTEYTGPESYVAEMIRERNLDWFPRMRA

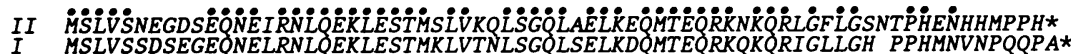

2679

2701

Fig. 3. Alignment of the amino acid sequence of the rat type $2 \mathrm{InsP}_{3}$ receptor (top line) with that of the type 1 receptor (second line, from Mignery et al. 1990) and the partial sequence of the putative human type $3 \mathrm{InsP}_{3}$ receptor (third line; C.L.Newton, G.A.Mignery and T.C.Südhof, in preparation). Identical residues are marked by dots above the sequence. Sequences are shown in single letter code and are numbered to the right. Amino acids belonging to putative transmembrane regions are underlined and the transmembrane regions are labeled M1 to M8. The position of the stop codon is indicated by an asterisk. 


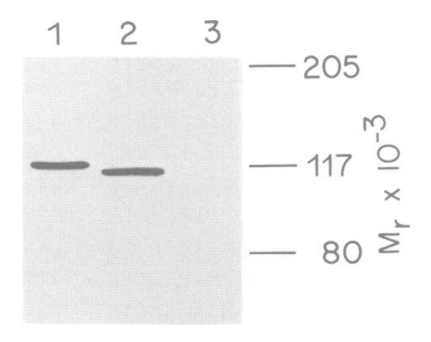

Fig. 4. Immunoblot analysis of proteins specified by the aminoterminal sequences of the type 1 and type 2 receptors expressed in COS cells. The amino-terminal 1081 and 1078 residues of the type 1 and type $2 \mathrm{InsP}_{3}$ receptors, respectively, were cloned into an expression vector fused to a sequence encoding the last 12 amino acids of the $116 \mathrm{~K}$ proton pump subunit. Cytosol from COS cells transfected with the type $1 \mathrm{InsP}_{3}$ receptor expression construct $\left(\mathrm{pIP}_{3} \mathrm{R}\right.$-Stop1081, lane 1), type $2 \mathrm{InsP}_{3}$ receptor construct ( $\mathrm{pIP}_{3} \mathrm{R} 2$-Stop1078, lane 2 ) or with control DNA (salmon sperm DNA, lane 3) were analyzed by immunoblotting using an antibody against their common carboxyterminal epitope followed by a peroxidase-labeled secondary antibody. Expression of the constructs results in soluble receptor proteins containing the full-length binding sites of the two $\mathrm{InsP}_{3}$ receptors and ending in the same carboxy-terminal sequence.

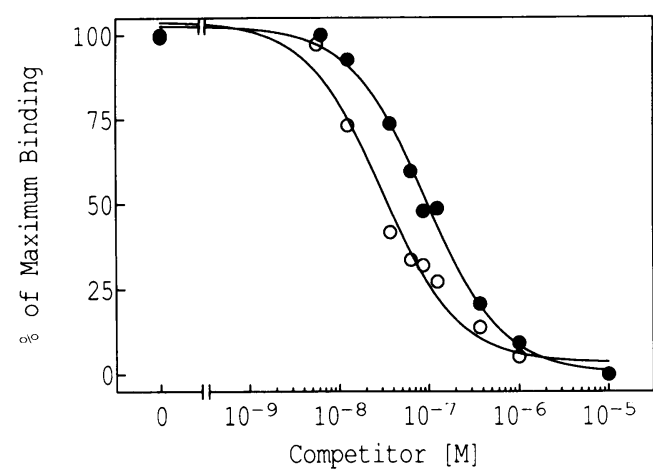

Fig. 5. Determination of the binding affinities of the ligand binding domains of the type 1 and type 2 InsP $\mathrm{P}_{3}$ receptors. Cytosol from COS cells transfected with constructs encoding the ligand binding domains of the type 1 (closed symbols) and type $2 \mathrm{InsP}_{3}$ receptors (open symbols) were used in binding-competition assays whereby the displacement of $\left.3.3 \mathrm{nM}{ }^{32} \mathrm{P}\right] \mathrm{InsP}_{3}$ by unlabeled $\mathrm{InsP}_{3}$ was determined. The line through the points represents the best fit to the data calculated using GraphPAD version 3.1 software, resulting in the determination of affinities for the type 1 and type 2 receptors of $89.5 \mathrm{nM}$ and of $27.0 \mathrm{nM}$, respectively. The experiment was repeated twice with similar results. COS cells transfected with control DNA showed no measurable Ins $\mathrm{P}_{3}$ binding (Table I).

Table I. Binding specificities of type 1 and type $2 \operatorname{Ins}(1,4,5) \mathrm{P}_{3}$ receptors

\begin{tabular}{lccccc}
\hline Competitor $(10 \mu \mathrm{M})$ & $\begin{array}{l}\mathrm{pIP}{ }_{3} \mathrm{R}-\mathrm{Stop} 1081 \\
\left(\text { c.p.m. } / \mathrm{mg} \times 10^{-3}\right)\end{array}$ & $\begin{array}{c}\text { Percentage of control } \\
\end{array}$ & $\begin{array}{l}\mathrm{pIP}_{3} \mathrm{R} 2-\mathrm{Stop} 1078 \\
\left.\text { (c.p.m./mg } \times 10^{-3}\right)\end{array}$ & Percentage of control Salmon sperm DNA \\
\hline- & $22.86 \pm 1.36$ & 100.0 & $11.33 \pm 0.59$ & 100.0 & $0.00 \pm 0.17$ \\
Inositol 1,4-bisphosphate & $22.86 \pm 1.17$ & 100.0 & $13.61 \pm 0.50$ & 120.1 & - \\
Inositol 4,5-bisphosphate & $16.03 \pm 0.47$ & 70.1 & $7.71 \pm 0.31$ & 68.0 & - \\
Inositol 1,4,5-trisphosphate & $0.00 \pm 0.18$ & 0.0 & $0.00 \pm 0.15$ & 0.0 & $0.00 \pm 0.96$ \\
Inositol 2,4,5-trisphosphate & $0.45 \pm 1.74$ & 2.0 & $0.60 \pm 0.48$ & 5.3 & - \\
Inositol 1,3,4,5-tetrakisphosphate & $8.68 \pm 1.20$ & 38.0 & $5.05 \pm 0.93$ & 44.6 & - \\
Inositol 1,4,5,6-tetrakisphosphate & $20.13 \pm 1.06$ & 88.1 & $11.59 \pm 0.55$ & 102.3 & - \\
Inositol 1,3,4,5,6-pentakisphosphate & $12.17 \pm 1.29$ & 53.2 & $7.22 \pm 0.61$ & 63.7 & - \\
Inositol hexakisphosphate & $17.86 \pm 0.49$ & 78.1 & $9.46 \pm 1.11$ & 83.5 & - \\
Heparin $(5 \mu \mathrm{g} / \mathrm{ml})$ & $2.62 \pm 0.94$ & 11.5 & $3.58 \pm 1.28$ & 31.6 & - \\
Heparin $(100 \mu \mathrm{g} / \mathrm{ml})$ & $0.00 \pm 0.50$ & 0.0 & $0.00 \pm 0.78$ & 0.0 & - \\
\hline
\end{tabular}

${ }^{3} \mathrm{H}$-Ins $\mathrm{P}_{3}$-binding $(25 \mathrm{nM}$ ) was measured with $50 \mu \mathrm{g}$ protein of the cytosol of COS cells transfected with the indicated DNAs. Quantitative immunoblotting showed that the expression of the $\mathrm{pIP}_{3} \mathrm{R}$-Stop1078 was $77 \%$ of that of $\mathrm{pIP}_{3} \mathrm{R}$-Stop1081 $\left(2.95\right.$ and $3.81 \times 10^{6}$ c.p.m. ${ }^{125}$ I-labeled antibody binding per $\mathrm{mg}$ protein, respectively) with no measurable InsP $\mathrm{P}_{3}$ binding or receptor expression in COS cells transfected with control DNA.

\section{Discussion}

In this study we have isolated and sequenced a set of overlapping cDNA clones encoding a novel $\mathrm{InsP}_{3}$ receptor. The complete primary structure of the new receptor was determined and consisted of 2701 amino acids and was found to be homologous over its entire length to the cerebellar $\mathrm{InsP}_{3}$ receptor-the only $\mathrm{InsP}_{3}$ receptor previously characterized. Expression of the amino-terminal domains of the novel receptor (referred to as type $2 \mathrm{InsP}_{3}$ receptor) and of the cerebellar receptor (referred to as type 1 receptor) in COS cells demonstrates that both bind $\mathrm{InsP}_{3}$ with high affinity and similar specificities, although the type 2 receptor has a significantly higher affinity than the cerebellar type 1 receptor. Together our results demonstrate the presence of different types of InsP $\mathrm{P}_{3}$ receptors in brain tissue, whose sequences and properties suggest that they may have different $\mathrm{Ins}_{3}$ binding affinities and regulatory characteristics.

Alignment of the sequences of the type 1 and type 2 $\mathrm{Ins}_{3}$ receptors reveals a scattered distribution of identical and diverse sequences with an overall sequence homology of $69 \%$. The structural design of the two $\mathrm{InsP}_{3}$ receptors is similar, suggesting that they are comprised of similar 3204 functional domains. We have previously proposed a domain model for the cerebellar $\mathrm{InsP}_{3}$ receptor that divides its sequence into a ligand binding domain, a coupling domain transducing the ligand binding signal, and a $\mathrm{Ca}^{2+}$ channel domain (Mignery and Südhof, 1990). Analysis of the sequence similarity between the receptors as a function of these domains suggests the ligand binding site is the most conserved region between the two receptors (Figure 6). This agrees well with the similar binding characteristics of the two receptors and suggests that their primary functional differences may be localized to the coupling domain and the $\mathrm{Ca}^{2+}$ channel domain.

The coupling domain separating the ligand binding domain from the putative channel domain is the least similar domain between the two types of receptors. The coupling domain contains the cAMP-dependent phosphorylation sites of the cerebellar $\mathrm{InsP}_{3}$ receptor, suggesting that it is the principal target of regulatory signals in the $\mathrm{InsP}_{3}$ receptor (Mignery et al., 1990; Ferris et al., 1991b). The lack of conservation between the two types of $\mathrm{InsP}_{3}$ receptors in this region suggests that the two receptors may be subject to different types of regulation. In addition, significant sequence 


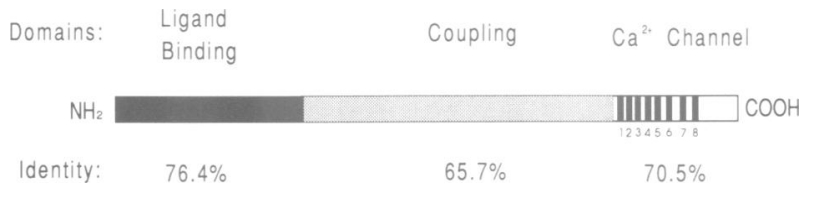

Fig. 6. Domain model of the InsP $\mathrm{P}_{3}$ receptors and sequence identities between the type 1 and type 2 Ins $\mathrm{P}_{3}$ receptors in the different domains. The three principal domains of the $\operatorname{InsP}_{3}$ receptor are described on top (Mignery and Südhof, 1990). The eight putative transmembrane regions in the carboxy-terminal fourth of the receptors are numbered and their position indicated by vertical lines. The sequence identity between the two types of $\operatorname{InsP}_{3}$ receptors in each domain is shown on the bottom.

differences are observed in the putative $\mathrm{Ca}^{2+}$ channel domain, particularly in the loops separating transmembrane regions. These differences suggest that the $\mathrm{Ca}^{2+}$ gating characteristics of the two receptors may also be different.

Our results demonstrate the presence of multiple types of $\mathrm{Ins}_{3}$ receptors co-expressed in brain. Many of the proteins involved in signal transduction at the cell surface have been shown to be present in multiple isoforms with different regulatory properties but this is the first such demonstration for a protein functioning downstream of the generation of Ins $\mathrm{P}_{3}$. What is the biological relevance of the presence of different types of InsP $\mathrm{P}_{3}$ receptors? We would like to suggest three major hypotheses that are not mutually exclusive and are based on examples of differentially regulated isoforms of proteins involved in signal transduction cascades.

1. Different types of $\operatorname{InsP}_{3}$ receptors may be functionally similar but have different regulatory properties. This would result in differences of the properties of intracellular $\mathrm{Ca}^{2+}$ stores dependent on which $\mathrm{InsP}_{3}$ receptors are expressed. This hypothesis is supported by the fact that the putative coupling domains of the $\operatorname{InsP}_{3}$ receptors that connect their ligand binding sites to the transmembrane regions is the least conserved between the receptor forms, suggesting that they may indeed be subject to differential regulation.

2. Different $\mathrm{InsP}_{3}$ receptors could have different intracellular functions specified by different intracellular localizations. Currently it seems unlikely, although not excluded, that an $\mathrm{InsP}_{3}$ receptor might be present in a subcellular membrane other than the endoplasmic reticulum, such as the plasma membrane (Penner et al., 1988). It is more likely that there are specialized subcompartments of the endoplasmic reticulum which may contain differentially regulated $\mathrm{Ca}^{2+}$ stores (Lechleiter et al., 1991; Villa et al., 1991).

3. Different types of InsP $\mathrm{P}_{3}$ receptors could have different intracellular functions analogous to the two forms of the ryanodine receptor. Ryanodine receptors, similar to Ins $\mathrm{P}_{3}$ receptors, release $\mathrm{Ca}^{2+}$ from intracellular stores. The ryanodine receptors from cardiac and skeletal muscle differ from each other in the coupling between membrane depolarization to $\mathrm{Ca}^{2+}$ release, and their sequences are 65\% identical (Takeshima et al., 1989; Otsu et al., 1990; Zorzato et al., 1990). It is possible that of the different types of InsP $\mathrm{P}_{3}$ receptors, one could be autonomous in the cell interior whereas the other similar to the skeletal muscle ryanodine receptor could be coupled to the plasma membrane. The low abundance of the type $2 \operatorname{InsP}_{3}$ receptor would support such a model.

All these hypotheses (independent of which will be proved to be correct) imply that $\mathrm{Ca}^{2+}$ signaling induced by $\operatorname{InsP}_{3}$ is much more complex than that envisioned by a single type of receptor. Clearly $\mathrm{Ca}^{2+}$-release from intracellular stores is not a uniform process but dependent on receptor types as well as secondary regulatory events.

\section{Materials and methods}

\section{cDNA cloning and DNA sequencing}

A rat cDNA library was screened as described (Südhof et al., 1987; Mignery et al., 1990) with an oligonucleotide complementary to the sequence of the last putative transmembrane region of both the ryanodine and the $\mathrm{InsP}_{3}$ receptors (oligonucleotide sequence: CAGCTGCAGGACGATGATGATGACCATGAAGAAGAA). Of the 15 clones isolated, most encoded the cerebellar $\mathrm{InsP}_{3}$ receptor but two clones upon sequencing were found to be distinct from but homologous to the cerebellar InsP $\mathrm{P}_{3}$ receptor. Although both of these clones terminated in poly(A) tails, one clone had a $1.9 \mathrm{~kb}$ longer $3^{\prime}$ untranslated region than the other, suggesting differential use of polyadenylation signals. The two clones were fully sequenced and further $5^{\prime}$ clones were isolated using oligonucleotides based on the sequences of the $5^{\prime}$ ends of these clones. The complete receptor was cloned in this manner on nine overlapping cDNA clones, several of which were isolated more than one time.

Polymerase chain reaction cloning of InsP $\mathrm{P}_{3}$ receptor related transcripts from a human kidney library was performed as described (Perin et al., 1991) using the oligonucleotide described above as the specific primer and primers from the flanking sequences of the vector as the second primer. Only two transcripts with homology to the cerebellar Ins $\mathrm{P}_{3}$ receptor were isolated, one of which was the human homologue of the cerebellar receptor, whereas the second encoded a novel sequence. DNA sequencing was performed by the chain termination method (Sanger et al., 1977) either manually using ${ }^{32} \mathrm{P}$ - and ${ }^{35} \mathrm{~S}$-labeled nucleotides or automatically on an ABI $370 \mathrm{~A}$ sequencer using single-stranded M13 subclones of the cDNA clones. Sequences were analyzed on an IBM-AT computer using Microgenie software and searched against GenBank release 64 and NBRF release 25, with no significant homology observed with any sequences in the databanks except for the $\mathrm{InsP}_{3}$ receptor and ryanodine receptor.

\section{Expression of the ligand binding sites of type 1 and type 2} Ins $P_{3}$ receptors by transfection

$\mathrm{pIP}_{3} \mathrm{R} 2$-Stop1078 is a mammalian expression vector in which the cytomegalovirus promoter drives the expression of the first 1078 amino acids of the type- $2 \mathrm{InsP}_{3}$ receptor. This sequence is followed by the 12 carboxy-terminal acids of the $116 \mathrm{~K}$ subunit of the proton pump (Perin et al., 1991) against which we obtained an antipeptide antibody that was used both to visualize and to quantify expression. $\mathrm{pIP}_{3} 2$-Stop 1078 was constructed by cloning the $2.45 \mathrm{~kb} E c o \mathrm{RI}-K p n \mathrm{I}$ fragment from p567-13 into pCMV2 (kind gift of Dr D.W.Russell, University of Texas Southwestern Medical Center, Dallas), followed by the $1.04 \mathrm{~kb} K p n I-P s t I$ fragment from pI 71 and by an oligonucleotide encoding the carboxy-terminal epitope. The corresponding type-1 Ins $\mathrm{P}_{3}$ receptor expression vector $\mathrm{pIP}_{3} \mathrm{R}$-Stop1081 was described previously (Mignery and Südhof, 1990). Purified DNA from both vectors was transiently transfected into $\operatorname{COS}$ cells and expression was analyzed by immunoblotting using peroxidase-labeled secondary antibodies and quantified using iodinated secondary antibodies and an Ambis radioanalytic imaging system. The cytosol from transfected cells was prepared as described (Mignery and Südhof, 1990) and used for binding measurements. All binding measurements were performed using the PEG precipitation assay (Chadwick et al., 1990) and tritiated InsP $_{3}$ (17 $\mathrm{Ci} /$ mmole) (NEN-Du Pont) except for the assays used for the determination of the binding affinities in which ${ }^{32} \mathrm{P}$-labeled $\mathrm{InsP}_{3}(155 \mathrm{Ci} / \mathrm{mmole})$ was used because of the required higher sensitivity. $\operatorname{COS}$ cells transfected with salmon sperm DNA were used as negative controls in all experiments. Binding data were evaluated and affinities calculated using GraphPAD InPlot version 3.1 software.

\section{RNA blotting experiments}

Total RNA was isolated from rat tissues and used for RNA-blots as described (Perin et al., 1986). All blots were probed with uniformly labeled singlestranded DNA probes generated on M13 templates, and washed at high stringencies.

\section{Acknowledgements}

We would like to thank I.Leznicki and K.Robinson for excellent technical assistance, and Drs M.S.Brown, J.L.Goldstein, P.De Camilli and D.W.Russell for stimulating discussions. This work was supported by NIH grants RO1 MH47510 and RO1 HL39644 and by the Howard Hughes Medical Institute. 


\section{References}

Berridge,M.J. (1990) J. Biol. Chem., 265, 9583-9586.

Berridge,M.J. and Irvine,R.F. (1989) Nature, 341, 197-205.

Chadwick,C.C., Saito,A. and Fleischer,S. (1990) Proc. Natl. Acad. Sci. USA, 87, 2132-2136.

De Camilli,P., Takei,K., Mignery,G.A. and Südhof,T.C. (1990) Nature, 344, 495.

Ely,J.A., Hunyady,L., Baukal,A.J. and Catt,K.J. (1990) Biochem. J., 268. 333-338.

Ferris,C.D., Cameron,A.M., Bredt,D.S., Huganir,R.L. and Snyder,S.H. (1991a) Biochem. Biophys. Res. Comm., 175, 192-198.

Ferris,C.D., Huganir,R.L., Bredt,D.S., Cameron,A.M. and Snyder,S.H. (1991b) Proc. Natl. Acad. Sci. USA, 88, 2232-2235.

Furuichi,T., Yoshikawa,S., Miyawaki,A., Wada,K., Maeda,N. and Mikoshiba,K. (1989) Nature, 342, 32-38.

Guillemette,G., Balla,T., Baukal,A.J. and Catt,K.J. (1988) J. Biol. Chem., 263, $4541-4548$.

Harootunian,A.T., Kao,J.P.Y., Paranjape,S., Adams,S.R., Potter,B.V.L. and Tsien,R.Y. (1991) Cell Calcium, 12, 153-164.

Irvine,R.F. (1990) FEBS Lett., 263, 5-9.

Kozak,M. (1989) J. Cell Biol., 108, 229-241

Lechleiter,J., Girard,S., Clapham,D. and Peralta,E. (1991) Nature, 350, 505-508.

Mignery,G.A. and Südhof,T.C. (1990) EMBO J., 9, 3893-3898.

Mignery,G.A., Südhof,T.C., Takei,K. and De Camilli,P. (1989) Nature, 342, $192-195$.

Mignery,G.A., Newton,C.L., Archer,B.T., III and Südhof,T.C. (1990) J. Biol. Chem., 265, 12679-12685.

Otsu,K., Willard,H.F., Khanna,V.K., Zorzato,F., Green and MacLennan,D.H. (1990) J. Biol. Chem., 265, 13472-13483.

Palmer,S. and Wakelam,M.J.O. (1989) Biochem. J., 260, 593-596.

Penner,R., Matthews,G. and Neher,E. (1988) Nature, 334, 499-504.

Perin,M.S., Fried,V.A., Slaughter,C.A. and Südhof,T.C. (1988) EMBO J., 7, 2697-2703.

Perin,M.S., Fried,V.A., Stone,D.K., Xie,X.-S. and Südhof,T.C. (1991) J. Biol. Chem., 266, 3877-3881.

Petersen,O.H., Gallacher,D.V., Wakui,M., Yule,D.I., Petersen,C.C.H. and Toescu,E.C. (1991) Cell Calcium, 12, 135-144.

Pietri,F., Hilly,M. and Mauger,J.-P. (1990) J. Biol. Chem., 265 , $17478-17485$.

Ross,C.A., Meldolesi,J., Milner,T.A., Satoh,T., Supattapone,S. and Snyder,S.H. (1989) Nature, 339, 468-470.

Ross,C.A., Bredt,D. and Snyder,S.H. (1990) Trends Neurosci., 13, 216-222.

Rossier,M.F., Capponi,A.M. and Vallotton,M.B. (1989) J. Biol. Chem., 264, $14078-14084$.

Sanger,F., Nicklen,S. and Coulson,A.R. (1977) Proc. Natl. Acad. Sci. USA, 74, 5463-5467.

Shears,S.B. (1991) Cancer Cells, 3, 97-99.

Streb,H., Irvine,R.F., Berridge,M.J. and Schulz,I. (1983) Nature, 306, 67-69.

Südhof,T.C., Lottspeich,F., Greengard,P., Mehl,E. and Jahn,R. (1987) Science, 238, 1142-1144.

Takei,K., Stukenbrok,H., Metcalf,A., Mignery,G.A., Südhof,T.C., Volpe,P. and De Camilli,P. (1992) J. Neurosci., in press.

Takeshima,H., Nishimura,S., Matsumoto,T., Ishida,H., Kangawa,K. Minamino,N., Matsuo,H., Ueda,M., Hanaoka,M., Hirose,T. and Numa,S. (1989) Nature, 339, 439-445.

Villa,A., Podini,P., Clegg,D.O., Pozzan,T. and Meldolesi,J. (1991) J. Cell Biol., 113, 779-791.

Woods,N.M., Cuthbertson,K.S.R. and Cobbold,P.H. (1986) Nature, 319, 600-602.

Zorzato,F., Fujii,J., Otsu,K., Phillips,M., Green,N.M., Lai,F.A., Meissner,G. and MacLennan,D.H. (1990) J. Biol. Chem., 265, 2244-2256.

Received on July 8, 1991; revised on August 1, 1991 